\title{
High-fat diet increases autophagic flux in pancreatic beta cells in vivo and ex vivo in mice
}

\author{
Kwan Yi Chu ${ }^{1}$ - Liam O'Reilly ${ }^{1}$ - Georg Ramm ${ }^{2}$ - Trevor J. Biden ${ }^{1,3}$
}

Received: 18 February 2015 / Accepted: 27 May 2015 /Published online: 14 June 2015

(C) Springer-Verlag Berlin Heidelberg 2015

\begin{abstract}
Aims/hypothesis Defective beta cell function during lipid oversupply and type 2 diabetes is associated with dysregulation of lysosomal function and autophagy. Whether this dysregulation represents augmentation or inhibition is unclear because of technical limitations in assaying autophagy. The current aim was to determine the effects of high-fat feeding on true autophagic flux in beta cells in vivo in mice, and to establish the relationship between autophagy, endoplasmic reticulum (ER) stress and apoptosis.

Methods Green fluorescent protein-microtubule-associated protein 1 light chain 3 (GFP-LC3) mice were fed chow or high-fat diets for 8-10 weeks and injected with $100 \mathrm{mg} \mathrm{kg}^{-1}$ day $^{-1}$ chloroquine for 5 days, prior to being killed, to block clearance of autophagic markers. Pancreases and livers were fixed and GFP-LC3 aggregates or autophagosomes were detected by fluorescence or electron microscopy, respectively. Independently, islets isolated from chow or high-fat-fed mice were treated for $2 \mathrm{~h}$ with chloroquine ex vivo, and immunoblotting was performed for markers of autophagy (LC3 lipidation - LC3II and p62/SQSTM1), ER stress (C/EBP
\end{abstract}

Electronic supplementary material The online version of this article (doi:10.1007/s00125-015-3665-x) contains peer-reviewed but unedited supplementary material, which is available to authorised users.

Kwan Yi Chu

k.chu@garvan.org.au

1 Diabetes and Metabolism Division, Garvan Institute of Medical Research, 384 Victoria Street, Darlinghurst, NSW 2010, Australia

2 Department of Biochemistry and Molecular Biology, Monash University, Clayton, VIC, Australia

3 St Vincent's Clinical School, Faculty of Medicine, The University of New South Wales, Sydney, NSW, Australia homology protein [CHOP], phosphorylated eukaryotic initiation factor $2 \alpha[\mathrm{p}$-eIF $\alpha]$ and inositol requiring enzyme $1 \alpha[\mathrm{p}$ IRE1 $\alpha]$ ) and apoptosis (cleaved caspase-3).

Results Numbers of autophagosomes and GFP puncta were increased in beta cells by combined high-fat feeding and chloroquine injection, indicative of enhanced autophagic flux. By contrast, GFP puncta were attenuated in liver under the same conditions. Relative to chow-fed controls, islets isolated from fat-fed mice exhibited higher LC3II levels when treated ex vivo with chloroquine. The combination of high-fat feeding and acute chloroquine treatment induced CHOP, p-eIF $2 \alpha$ and caspase-3, but not either treatment alone.

Conclusions/interpretation We provide the first in vivo demonstrations that high-fat feeding increases autophagic flux in pancreatic beta cells, and that this serves to protect against induction of terminal ER stress. We also highlight an approach for monitoring dietary alterations in autophagic flux using ex vivo manipulation of isolated islets.

Keywords Autophagic flux · Autophagy $\cdot$ Chloroquine $\cdot$ ER stress · GFP-LC3 · High-fat diet · Lipid droplet · Liver · Pancreatic beta cell

$\begin{array}{ll}\begin{array}{l}\text { Abbreviations } \\ \text { CHOP }\end{array} & \text { C/EBP homology protein } \\ \text { eIF2 } \alpha & \text { Eukaryotic initiation factor } 2 \alpha \\ \text { ER } & \text { Endoplasmic reticulum } \\ \text { ESM } & \text { Electronic supplementary material } \\ \text { GFP-LC3 } & \begin{array}{l}\text { Green fluorescent protein-microtubule- } \\ \text { associated protein 1 light chain } 3\end{array} \\ \text { IRE1 } \alpha & \text { Inositol requiring enzyme } 1 \alpha \\ \text { ISR } & \text { Integrated stress response }\end{array}$




$\begin{array}{ll}\text { LC3 } & \begin{array}{l}\text { Microtubule-associated protein 1 light } \\ \text { chain 3 }\end{array} \\ \text { p62/SQSTM1 } & \text { Sequestosome 1 }\end{array}$

\section{Introduction}

(Macro)autophagy is a lysosomal degradation system for eliminating damaged organelles and long-lived or aggregated proteins. It is crucial for the function and survival of pancreatic beta cells [1]. Cargo destined for clearance is engulfed by a double membrane, thus forming an autophagosome, which then fuses with a lysosome to facilitate degradation. A key signalling step involves the microtubule-associated protein 1 light chain 3 (LC3). Activation facilitates the conversion of LC3I to LC3II, which binds to the autophagosome membrane [1].

Morphological studies of human or rodent islets show increased numbers of autophagosomes during type 2 diabetes and chronic lipid exposure (lipotoxicity) [2-6]. Although usually interpreted as enhanced autophagy, this might actually reflect the opposite since disruption of lysosomal function (and thus inhibition of distal autophagy) induces similar changes. Consistent with this possibility, gene expression studies suggest that beta cell lysosomal activity is inhibited during diabetes and lipotoxicity $[4,6]$.

In the current study we have employed in vivo [7] treatments in mice to inhibit lysosomal function, thereby facilitating analysis of true autophagic flux in islets. Autophagic flux was enhanced by high-fat feeding, which we also confirmed using islets treated ex vivo with lysosomal inhibitors. The ex vivo model also suggests that activation of autophagy limits induction of $\mathrm{C} / \mathrm{EBP}$ homology protein (CHOP), which couples to apoptosis downstream of both endoplasmic reticulum (ER) stress and the integrated stress response (ISR).

\section{Methods}

Animals All studies were approved by the Garvan/St Vincent's Hospital Animal Ethics Committee. We used C57BL/6 mice (ex vivo studies or electron microscopy) or homozygous green fluorescent protein (GFP)-LC3 transgenic mice (RIKEN, Wako, Saitama, Japan) [8] (fluorescence and electron microscopy). Eight-week-old male mice were fed ad libitum for 8-10 weeks on either a standard chow $(10.88 \mathrm{~kJ} / \mathrm{g}$; $8 \%$ fat, $21 \%$ protein and $71 \%$ carbohydrate) or a high-fat diet $(19.67 \mathrm{~kJ} / \mathrm{g} ; 45 \%$ fat, $20 \%$ protein and $35 \%$ carbohydrate). To estimate autophagic flux in vivo, mice were injected with the lysosomal inhibitor chloroquine (100 $\mathrm{mg} \mathrm{kg}^{-1}$ day $^{-1}$; SigmaAldrich, Sydney, NSW, Australia) or saline control daily for 5 days before being killed [7].
Islet isolation, treatment and immunoblotting Mouse islets were isolated as described previously [9] and then incubated with or without chloroquine $(50 \mu \mathrm{mol} / \mathrm{l})$ for $2 \mathrm{~h}$ immediately after isolation. Immunoblotting of islet lysates was as recently described [9]. For further details and sources of antibodies please refer to the electronic supplementary material (ESM) Methods.

Fluorescence and confocal microscopy and Oil Red $O$ staining Nonfasted liver or pancreas was harvested, fixed in $4 \%$ paraformaldehyde in PBS overnight, followed by $30 \%$ sucrose incubation at $4^{\circ} \mathrm{C}$ for $6 \mathrm{~h}$. For details of embedding, staining, mounting and antibodies please see ESM Methods.

Electron microscopy Isolated islets from chow or high-fatfed mice with 5 day chloroquine or PBS injection were immediately fixed in $3 \%$ glutaraldehyde in phosphate buffer $(0.05 \mathrm{~mol} / \mathrm{l}, \mathrm{pH} 7.2)$ and then post fixed in $1 \%$ osmium tetroxide and $2 \%$ uranyl acetate. After dehydration, the islet pellet was embedded in Epon resin (EM Sciences, Hatfield, PA, USA) for cutting of ultrathin sections. Ultrathin sections were stained with lead citrate and uranyl acetate before imaging using a Hitachi H-7500 transmission electron microscope at $80 \mathrm{kV}$ (Hitachi, Tokyo, Japan) equipped with a Gatan Multiscan 791 camera (Gatan, Pleasanton, CA, USA). To estimate the number of autophagosomes per beta cell profile, 40 profiles with a visible nucleus per conditions were examined under the electron microscope and all autophagosomal structures were counted.

RNA analysis Isolated islets were immediately lysed for total RNA extraction with the RNeasy Mini Kit (Qiagen, Melbourne, VIC, Australia). The extracted RNA was converted to complementary DNA (cDNA) and gene expression was quantified using the SYBR Green in 7900 Real Time PCR System (Applied Biosystems; Life Technologies Australia, Melbourne, VIC, Australia). The primer set for p62/Sqstm 1 was: (forward) 5'-GCTGCCCTATACCCACATCT-3' and (reverse) 5'-CGCCTTCATCCGAGAAAC-3'.

Statistical analysis All statistics were performed using GraphPad Prism 6 software (GraphPad software, La Jolla, CA, USA), as detailed in the figure legends.

\section{Results}

Autophagic flux is increased in pancreatic beta cells after high-fat feeding Autophagic flux was assessed in islets of GFP-LC3 mice by quantifying LC3II aggregation on the surface of autophagosomes following in vivo chloroquine injection $[7,8]$. Basal flux was low, with few fluorescent puncta observed in islets of chow-fed mice even following 
chloroquine treatment (Fig. 1a, b). Overall, fluorescence was augmented by high-fat feeding alone (Fig. 1c), but aggregation of GFP-LC3 occurred only in the presence of chloroquine (Fig. 1d). The number of puncta was significantly enhanced by this combined treatment vs each other group (Fig. 1e).

The puncta scoring protocol was verified in two ways. First, electron microscopy was employed to quantify autophagosomes directly (Fig. 1f, ESM Fig. 1). Although not reaching statistical significance, the trends across the treatment groups agreed very well with the GFP-LC3 data (shown in Fig. 1e, f). More importantly, the actual numbers were very similar using both techniques, suggesting that the puncta are truly autophagosomes. Second, we excluded the possibility of GFP-LC3 aggregating on the surface of lipid droplets [10], since these were not observed in pancreas sections under corresponding conditions (ESM Fig. 2).

Autophagic flux is reduced in liver after high-fat feeding In liver, fluorescent puncta were increased by chloroquine injection in chow-fed mice (ESM Fig. 3a,b) but reduced by high-fat feeding (ESM Fig. 3c,d). This finding suggests that the highfat diet reduced autophagic flux in the liver as previously suggested [10,11], which was confirmed with another marker, p62/SQSTM1 (sequestosome 1), which is cleared in lysosomes and thus correlates inversely with autophagy. p62/SQSTM1 staining in the liver was increased (consistent with reduced autophagic flux) by both chloroquine treatment and a high-fat diet (ESM Fig. 4). In pancreas, we also observed the expected increase in $\mathrm{p} 62 / \mathrm{SQSTM} 1$ due to chloroquine (ESM Fig. 5a,b,e). However, staining was not reduced by high-fat feeding (as would be predicted by enhanced autophagy) and actually increased in the combined high-fat/chloroquine group (ESM Fig. 5c,d,e). This discrepancy is potentially resolved by the observation that $p 62 / \mathrm{Sqstm} 1 \mathrm{mRNA}$ expression was also increased by high-fat feeding (ESM Fig. 5f), which would tend to counter any augmentation of protein clearance.

\section{Inhibition of autophagy but not high-fat-feeding increases}

ER stress To extend these findings to a simpler model, we isolated islets from chow or high-fat-fed mice and treated them ex vivo with chloroquine for $2 \mathrm{~h}$. The autophagic markers LC3II and p62/SQSTM1, as well as cleaved caspase-3 (an indicator of apoptosis) were significantly elevated only in the combined treatment groups (Fig. 2a-c). CHOP expression and eukaryotic initiation factor $2 \alpha$ (eIF $2 \alpha$ ) phosphorylation, respectively, terminal and proximal markers of both ER stress and the ISR, were unaltered by high-fat feeding alone, but were increased when combined with chloroquine (Fig. 2d, e). This pattern matched less well with phosphorylation of inositol requiring enzyme $1 \alpha$ (IRE1 $\alpha$; Fig. 2f), a more selective indicator of ER stress. These results suggest that inhibition of distal autophagy with chloroquine exacerbates the ISR. Conversely, the activation of
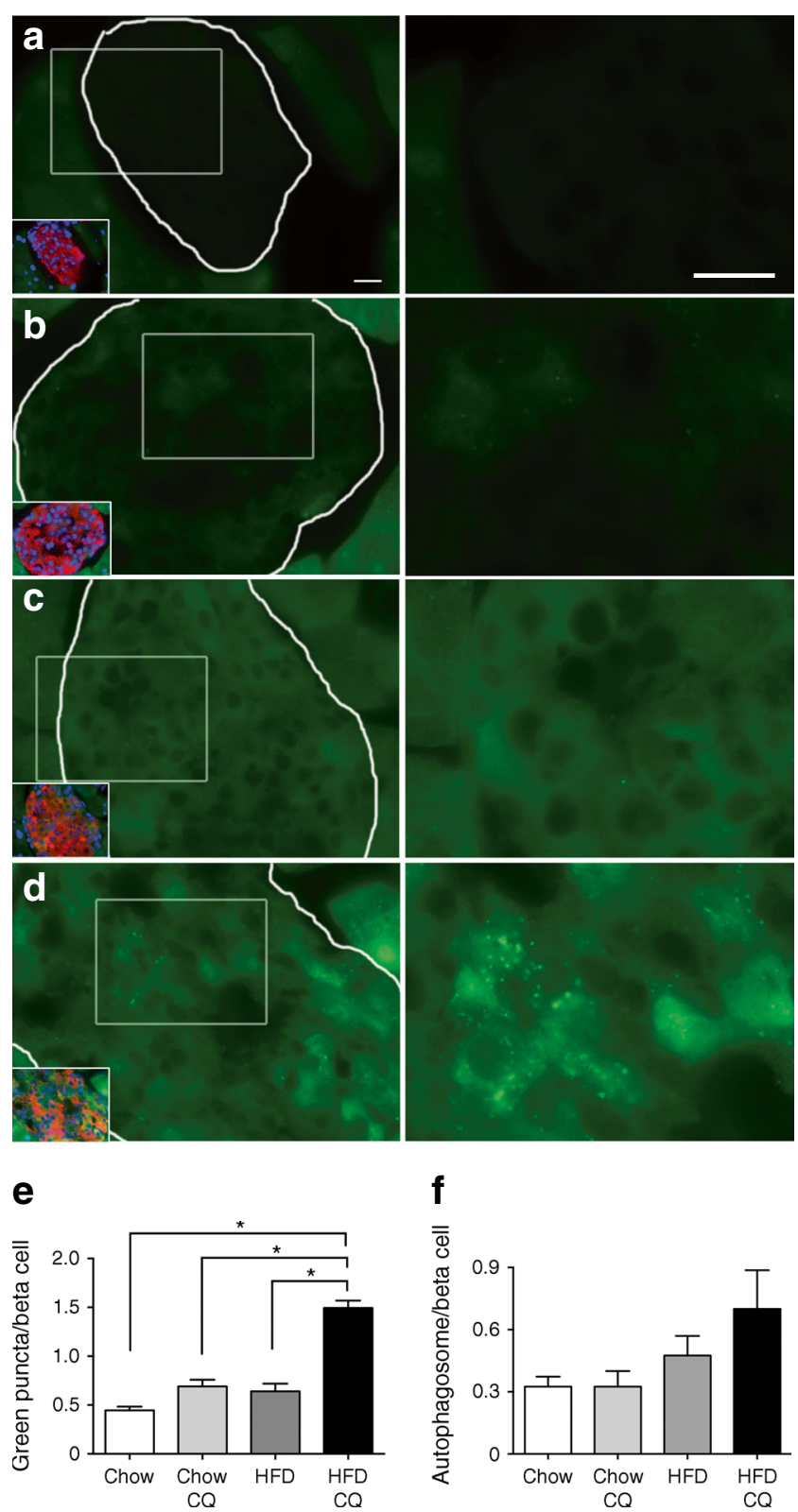

Fig. 1 Autophagic flux (GFP-LC3 puncta) is increased by high-fat feeding in mouse pancreatic beta cells. The GFP-LC3 signal is shown in green, beta cells are labelled with insulin in red and nuclei are labelled with DAPI in blue. Representative images of GFP-LC3 signal in pancreatic sections (islet marked by white line) from mice on (a) chow diet, (b) chow diet with chloroquine (CQ) injection, (c) high-fat diet (HFD) and (d) HFD with CQ injection. Scale bar, $10 \mu \mathrm{m}$. Insets show the merged colour images. Magnified images of areas indicated with white boxes are provided in the right-hand column. (e) The number of puncta per beta cell was counted in at least ten islets from each mouse. The data are indicated as mean \pm SEM of four to seven mice for each group. Kruskal-Wallis oneway ANOVA tests were used with Dunn's test for multiple comparisons of groups. ${ }^{*} p<0.05$ for the difference between the indicated groups. (f) The number of autophagic structures visible across 40 profiles from each treatment condition analysed under a transmission electron microscope was quantified. Results are mean $\pm \mathrm{SEM}$ of four mice per group, with the HFD plus CQ group differing from the chow diet group $(p=0.11)$ analysed by the Mann-Whitney $U$ test 
a

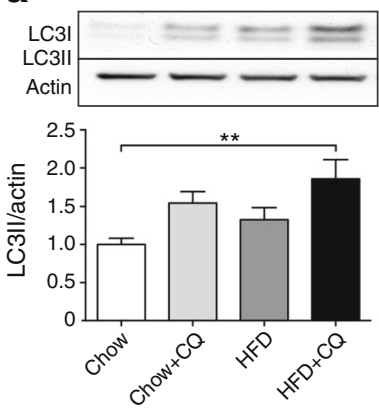

C

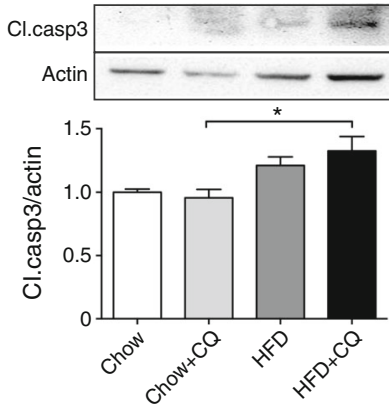

e
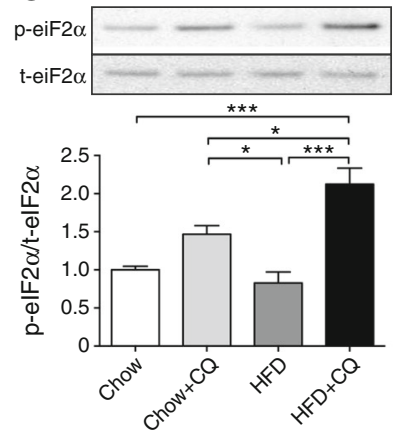

b

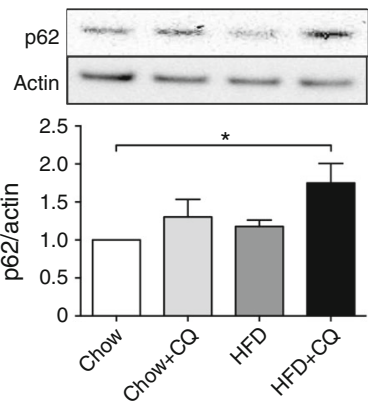

d

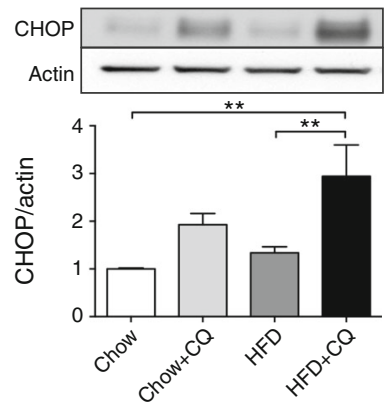

f
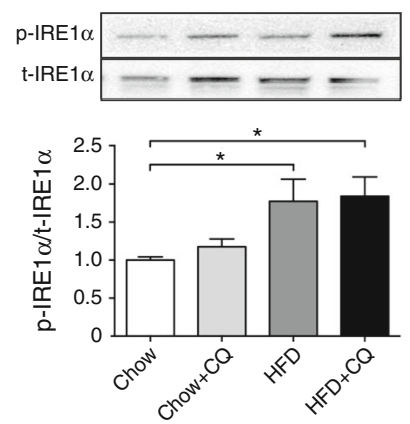

Fig. 2 Effect of high-fat feeding and lysosomal inhibition by chloroquine on isolated mouse islets. Islets were isolated from GFP-LC3 mice after chow or high-fat diet (HFD) and incubated with or without $50 \mu \mathrm{mol} / 1$ chloroquine (CQ) in RPMI medium containing $11.1 \mathrm{mmol} / 1$ glucose for $2 \mathrm{~h}$. Markers for autophagy (LC3II), apoptosis (cleaved caspase 3) and ER stress (CHOP, p-eIF2 $\alpha$ and p-IRE1 $\alpha$ ) were analysed by western blot. Representative gels and quantifications (normalised to loading controls) of (a) LC3II, (b) p62/SQSTM1, (c) cleaved caspase 3, (d) CHOP, (e) $p$-eIF2 $\alpha$ and (f) p-IRE1 $\alpha$ are presented. The data are indicated as mean \pm SEM of eight mice for each group. One-way ANOVA and the Tukey post hoc test were used for multiple comparisons of groups. ${ }^{*} p<0.05,{ }^{* *} p<0.01$ and $* * * p<0.001$ for the difference between the indicated groups. Cl.casp3, cleaved caspase 3

autophagy by high-fat feeding would normally help counter the ISR.

\section{Discussion}

Our study provides three main advances, two conceptual and one technical. Primarily, we provide the first direct evidence

that high-fat feeding stimulates autophagic flux in beta cells in vivo. This has been previously inferred from electron microscopy studies demonstrating increases in autophagosomes and vacuole formation [2], consistent with analyses of islets from $d b / d b$ mice [2], patients with diabetes [6] and in vitro models of beta cell lipotoxicity [3-5]. However, interpretation of these morphological changes is problematic because an accumulation of autophagosomes could result from impaired lysosomal clearance rather than an enhancement of upstream autophagy. Indeed, downregulated expression of some lysosomal enzymes in islets from patients with diabetes would be consistent with this possibility $[4,6]$.

To overcome these drawbacks we have incorporated the crucial intervention of injecting chloroquine into mice which, by inhibiting clearance of autophagosomes, allows for estimation of true autophagic flux [7]. By use of this protocol we showed that basal autophagy in beta cells is relatively low but augmented following high-fat feeding. This result was demonstrated by both electron microscopy and using the GFPLC3 mouse model, with excellent quantitative and qualitative concordance between the two approaches. Although LC3 can also associate with lipid droplets [10] these were not detected in beta cells under conditions in which GFP-LC3 puncta were readily observable. The specificity of our approach is further underscored by the contrast with liver, where autophagic flux was inhibited by high-fat feeding, consistent with previous work $[10,11]$.

Our second key finding is that inhibition of autophagy in beta cells unmasks an induction of CHOP due to high-fat feeding that has not been demonstrated previously in vivo [12]. Whether CHOP accumulation under these conditions arises downstream of some (eIF $2 \alpha$ ), but not all (IRE1 $\alpha$ ), arms of the unfolded protein response, or as a consequence of the ISR, was not resolved in our studies. Regardless, our data now suggest that suppression of CHOP would represent a beneficial adaptation to high-fat feeding, albeit one that might come at the cost of reduced insulin secretion due to disruption of lipid signalling pathways [9]. Although it is commonly assumed, based on genetic models $[12,13]$, that modulation of ER stress explains the reciprocal relationship between autophagy and beta cell apoptosis [2, 4, 14], we believe our CHOP data are the first to confirm this mechanistic link in the context of high-fat feeding. Our results would also tend to counter the idea, suggested by some in vitro studies [3] that lipotoxic ER stress is actually triggered by autophagy.

The third advance is validation of a method for evaluating autophagy ex vivo that accurately reflects flux in vivo. This method relies on acutely treating islets with chloroquine for $2 \mathrm{~h}$, which obviates the requirement for in vivo injections. LC3II has been previously analysed using islets from various models ex vivo, but in the absence of the additional incubation with chloroquine, definitive conclusions about flux are problematic. Our results also confirm prior findings that, although 
p62/SQSTM1 might be a useful marker of autophagic flux in some situations, in the context of beta cell lipotoxicity its interpretation is confounded by a countermanding stimulation of $p 62 / S q s t m 1$ gene expression [15].

In conclusion, our results suggest that enhanced autophagy in beta cells is an adaptation to high-fat feeding that helps prevent ER stress and/or the ISR. It will now be important to address whether this adaptation is defective in diabetes. Our study also highlights the need to elucidate mechanisms whereby fatty acids regulate autophagic signalling in beta cells, which appear to respond very differently to (for example) hepatocytes.

Acknowledgements We thank A. Costin (Monash University, Clayton, VIC, Australia) for islet processing and sectioning for electron microscopy, as well as R. Laybutt and G. Pearson (Garvan Institute of Medical Research, Darlinghurst, NSW, Australia) for critical review of the manuscript.

Funding This work was supported by a grant from the National Health and Medical Research Council of Australia (project grant 1051658) to TJB.

Duality of interest The authors declare that there is no duality of interest associated with this manuscript.

Contribution statement KYC acquired data and with TJB contributed to the conception and design of the experiments, interpretation of the data, and wrote and critically reviewed the article. LO and GR contributed to acquisition and analysis of data, and critical review of the article. All authors approved the final version of the manuscript. KYC is the guarantor of this work.

\section{References}

1. Lee MS (2014) Role of islet beta cell autophagy in the pathogenesis of diabetes. Trends Endocrinol Metab 25:620-627
2. Ebato C, Uchida T, Arakawa M et al (2008) Autophagy is important in islet homeostasis and compensatory increase of beta cell mass in response to high-fat diet. Cell Metab 8:325-332

3. Choi SE, Lee SM, Lee YJ et al (2009) Protective role of autophagy in palmitate-induced INS-1 beta-cell death. Endocrinology 150:126-134

4. Cnop M, Abdulkarim B, Bottu G et al (2014) RNA-sequencing identifies dysregulation of the human pancreatic islet transcriptome by the saturated fatty acid palmitate. Diabetes 63:1978-1993

5. Martino L, Masini M, Novelli M et al (2012) Palmitate activates autophagy in INS-1E beta-cells and in isolated rat and human pancreatic islets. PLoS One 7, e36188

6. Masini M, Bugliani M, Lupi R et al (2009) Autophagy in human type 2 diabetes pancreatic beta cells. Diabetologia 52:1083-1086

7. Iwai-Kanai E, Yuan H, Huang C et al (2008) A method to measure cardiac autophagic flux in vivo. Autophagy 4:322-329

8. Mizushima N, Yamamoto A, Matsui M, Yoshimori T, Ohsumi Y (2004) In vivo analysis of autophagy in response to nutrient starvation using transgenic mice expressing a fluorescent autophagosome marker. Mol Biol Cell 15:1101-1111

9. Pearson GL, Mellett N, Chu KY et al (2014) Lysosomal acid lipase and lipophagy are constitutive negative regulators of glucosestimulated insulin secretion from pancreatic beta cells. Diabetologia 57:129-139

10. Singh R, Kaushik S, Wang Y et al (2009) Autophagy regulates lipid metabolism. Nature 458:1131-1135

11. Liu HY, Han J, Cao SY et al (2009) Hepatic autophagy is suppressed in the presence of insulin resistance and hyperinsulinemia: inhibition of FoxO1-dependent expression of key autophagy genes by insulin. J Biol Chem 284:31484-31492

12. Biden TJ, Boslem E, Chu KY, Sue N (2014) Lipotoxic endoplasmic reticulum stress, beta cell failure, and type 2 diabetes mellitus. Trends Endocrinol Metab 25:389-398

13. Stienstra R, Haim Y, Riahi Y, Netea M, Rudich A, Leibowitz G (2014) Autophagy in adipose tissue and the beta cell: implications for obesity and diabetes. Diabetologia 57:1505-1516

14. Las G, Serada SB, Wikstrom JD, Twig G, Shirihai OS (2011) Fatty acids suppress autophagic turnover in beta-cells. J Biol Chem 286:42534-42544

15. Rivera JF, Gurlo T, Daval M et al (2011) Human-IAPP disrupts the autophagy/lysosomal pathway in pancreatic beta-cells: protective role of p62-positive cytoplasmic inclusions. Cell Death Differ $18: 415-426$ 